\title{
On 1-convexity and nucleolus of co-insurance games *
}

\author{
Theo S.H. Driessen ${ }^{\dagger} \quad$ Vito Fragnelli $^{\ddagger} \quad$ Ilya V. Katsev ${ }^{\S}$ \\ Anna B. Khmelnitskaya
}

\begin{abstract}
The situation, in which an enormous risk is insured by a number of insurance companies, is modeled through a cooperative TU game, the so-called coinsurance game, first introduced in Fragnelli and Marina (2004). In this paper we show that a co-insurance game possesses several interesting properties that allow to study the nonemptiness and the structure of the core and to construct an efficient algorithm for computing the nucleolus.
\end{abstract}

Keywords: cooperative game, insurance, core, nucleolus

Mathematics Subject Classification (2000): 91A12, 91A40, $91 \mathrm{~B} 30$

JEL Classification Number: C71

\section{Introduction}

In many practical situations the risks are too large to be insured by only one company, for example environmental pollution risk. As a result, several insurance companies share the liability and premium. In such a risk sharing situation two important practical questions arise: which premium the insurance companies have to charge and how should the companies split the risk and the premium keeping themselves as much competitive as possible and at the same time obtaining a fair division? In Fragnelli and Marina [8] the problem is approached from a game theoretic point of view through the construction of a cooperative game, the so-called co-insurance game. In this paper we study the nonemptiness and the structure of the core and the nucleolus of the co-insurance game subject to the premium value. If the premium is

\footnotetext{
${ }^{*}$ The research of Theo Driessen, Ilya Katsev, and Anna Khmelnitskaya was supported by NWO (The Netherlands Organization for Scientific Research) grant NL-RF 047.017.017. The research of Ilya Katsev was also supported by RFBR (Russian Foundation for Basic Research) grant 0906-00155. The research was partially done during Anna Khmelnitskaya 2008 research stay at the Tilburg Center for Logic and Philosophy of Science (TiLPS, Tilburg University) whose hospitality and support are highly appreciated as well.

${ }^{\dagger}$ University of Twente, Department of Applied Mathematics, P.O. Box 217, 7500 AE Enschede, The Netherlands, e-mail: t.s.h.driessen@ewi.utwente.nl

${ }^{\ddagger}$ University of Eastern Piedmont, Department of Science and Advanced Technologies, Viale T. Michel 11, 15121, Alessandria, Italy, e-mail: vito.fragnelli@mfn.unipmn.it

${ }^{\S} \mathrm{SPb}$ Institute for Economics and Mathematics Russian Academy of Sciences, 1 Tchaikovsky St., 191187 St.Petersburg, Russia, e-mail: katsev@yandex.ru

${ }^{\top} \mathrm{SPb}$ Institute for Economics and Mathematics Russian Academy of Sciences, 1 Tchaikovsky St., 191187 St.Petersburg, Russia, e-mail: a.khmelnitskaya@math.utwente.nl
} 
large enough, the core is empty. If the premium meets a critical upper bound, the nonemptiness of the core, being a single allocation composed of player's marginal contributions, turns out to be equivalent to the so-called 1-convexity property of the co-insurance game. Moreover, if nonemptiness applies, the co-insurance game inherits the 1-convexity property while lowering the premium till a critical lower bound induced by the individual evaluations of the enormous risk. In addition, 1-convexity of the co-insurance game yields the linearity of the nucleolus which, in particular, appears to be a linear function of the variable premium. If 1-convexity does not apply, then for the premium below another critical number we present an efficient algorithm for computing the nucleolus.

The interest to the class of co-insurance games is not only because they reflect the well defined actual economic situations but also it is determined by the fact that any arbitrary nonnegative monotonic cooperative game may be represented in the form of a co-insurance game. This allows to glance into the nature of a nonnegative monotonic game from another angle and by that to discover its new properties and peculiarities. Further, a co-insurance game appears to be a very natural extension of the well-known bankruptcy game introduced by Aumann and Maschler [2]. Besides, the study of 1-convex/1-concave TU games possessing a nonempty core and for which the nucleolus is linear was initiated by Driessen and Tijs [7] and Driessen [5], but until recently appealing abstract and practical examples of these classes of games were missing. The first practical example of a 1-concave game, the so-called library cost game, and the 1-concave complementary unanimity basis for the entire space of TU games were introduced in Driessen, Khmelnitskaya, and Sales [6]. A co-insurance game under some conditions provides a new practical example of a 1convex game. Moreover, in this paper we also show that a bankruptcy game is not only convex but 1-convex as well when the estate is sufficiently large comparatively to the given claims.

The structure of the paper is as follows. Basic definitions and notation are given in Sect. 2. Sect. 3 studies the nonemptiness and the structure of the core and the nucleolus of a co-insurance game with respect to the premium value. In Sect. 4 an algorithm for computing the nucleolus is introduced.

\section{Preliminaries}

Recall some definitions and notation. A cooperative game with transferable utility (TU game) is a pair $\langle N, v\rangle$, where $N=\{1, \ldots, n\}$ is a finite set of $n \geq 2$ players and $v: 2^{N} \rightarrow \mathbb{R}$ is a characteristic function, defined on the power set of $N$, satisfying $v(\emptyset)=0$. A subset $S \subseteq N$ (or $S \in 2^{N}$ ) of $s$ players is called a coalition, and the associated real number $v(S)$ represents the worth of the coalition $S$; in particular, $N$ is call a grand coalition. The set of all games with a fixed player set $N$ is denoted by $\mathcal{G}_{N}$ and it can be naturally identified with the Euclidean space $\mathbb{R}^{2^{n}-1}$. For simplicity of notation and if no ambiguity appears, we write $v$ instead of $\langle N, v\rangle$ when referring to a game. A value is an operator $\xi: \mathcal{G}_{N} \rightarrow \mathbb{R}^{n}$ that assigns to any game $v \in \mathcal{G}_{N}$ a vector $\xi(v) \in \mathbb{R}^{n}$; the real number $\xi_{i}(v)$ represents the payoff to the player $i$ in the game $v$. A payoff vector $x \in \mathbb{R}^{n}$ is said to be efficient in the game $v$, if $x(N)=v(N)$. Given a game $v$, the subgame $\left.v\right|_{T}$ with the player set $T \subseteq N$, $T \neq \emptyset$, is a game defined by $\left.v\right|_{T}(S)=v(S)$ for all $S \subseteq T$. A game $v$ is nonnegative 
if $v(S) \geq 0$ for all $S \subseteq N$. A game $v$ is monotonic if $v(S) \leq v(T)$ for all $S \subseteq T \subseteq N$. For the cardinality of a given set $A$ we use a standard notation $|A|$ along with lower case letters like $n=|N|, m=|M|, n_{k}=\left|N_{k}\right|$, and so on. We also use standard notation $x(S)=\sum_{i \in S} x_{i}$ and $x_{S}=\left\{x_{i}\right\}_{i \in S}$, for all $x \in \mathbb{R}^{n}, S \subseteq N$.

The imputation set of a game $v \in \mathcal{G}_{N}$ is defined as a set of efficient and individually rational payoff vectors

$$
I(v)=\left\{x \in \mathbb{R}^{n} \mid x(N)=v(N), x_{i} \geq v(i), \text { for all } i \in N\right\},
$$

while the preimputation set of a game $v \in \mathcal{G}_{N}$ is defined as a set of efficient payoff vectors

$$
I^{*}(v)=\left\{x \in \mathbb{R}^{n} \mid x(N)=v(N)\right\} .
$$

The core [9] of a game $v \in \mathcal{G}_{N}$ is defined as a set of efficient payoff vectors that are not dominated by any coalition, i.e.,

$$
C(v)=\left\{x \in \mathbb{R}^{n} \mid x(N)=v(N), x(S) \geq v(S), \text { for all } S \subseteq N\right\} .
$$

A game $v \in \mathcal{G}_{N}$ is balanced if $C(v) \neq \emptyset$.

For any game $v \in \mathcal{G}_{N}$, the excess of a coalition $S \subseteq N$ with respect to a vector $x \in \mathbb{R}^{n}$ is given by

$$
e^{v}(S, x)=v(S)-x(S) .
$$

The nucleolus [12] is a value defined as a minimizer of the lexicographic ordering of components of the excess vector of a given game $v \in \mathcal{G}_{N}$ arranged in weakly decreasing order of their magnitude over the imputation set $I(v)$.

The prenucleolus is a value defined as a minimizer of the lexicographic ordering of components of the excess vector of a given game $v \in \mathcal{G}_{N}$ arranged in weakly decreasing order of their magnitude over the preimputation set $I^{*}(v)$.

For a game $v \in \mathcal{G}_{N}$ with a nonempty core the nucleolus $\nu(v)$ belongs to $C(v)$.

For a game $v \in \mathcal{G}_{N}$ we consider the vector $m^{v} \in \mathbb{R}^{n}$ of marginal contributions to the grand coalition, the so-called marginal worth vector, defined as

$$
m_{i}^{v}=v(N)-v(N \backslash\{i\}), \quad \text { for all } i \in N,
$$

and the $g a p$ vector $g^{v} \in \mathbb{R}^{2^{\mathrm{N}}}$ defined as

$$
g^{v}(S)=\left\{\begin{array}{cc}
\sum_{i \in S} m_{i}^{v}-v(S), & S \subseteq N, S \neq \emptyset, \\
0, & S=\emptyset,
\end{array}\right.
$$

i.e., the gap vector measures for every $S \subseteq N$ the total coalitional surplus of marginal contributions to the grand coalition over its worth. In fact, $g^{v}(S)=-e^{v}\left(S, m^{v}\right)$, with $e^{v}\left(S, m^{v}\right)$ being th excess vector of $S$ in game $v$ at payoff vector $x=m^{v}$.

It is easy to check that in any game $v \in \mathcal{G}_{N}$, the vector $m^{v}$ relates to the core being an upper bound in that $x_{i} \leq m_{i}^{v}$, for any $x \in C(v)$ and all $i \in N$. In particular, the condition $v(N) \leq \sum_{i \in N} m_{i}^{v}$ is a necessary (but not sufficient) condition for nonemptiness of the core of the arbitrary game $v$, i.e., a strictly negative gap of the grand coalition $g^{v}(N)<0$ implies $C(v)=\emptyset$.

A game $v \in \mathcal{G}_{N}$ is convex if for all $i \in N$ and all $S \subseteq T \subseteq N \backslash\{i\}$,

$$
v(S \cup\{i\})-v(S) \leq v(T \cup\{i\})-v(T),
$$


or equivalently, if for all $S, T \subseteq N$,

$$
v(S)+v(T) \leq v(S \cup T)+v(S \cap T) .
$$

Any convex game has a nonempty core [13].

Proposition 1 For every convex game $v \in \mathcal{G}_{N}$ it holds that

$$
g^{v}(N) \geq 0, \quad \text { and } \quad g^{v}(N) \geq g^{v}(S), \quad \text { for all } S \subseteq N .
$$

Proof The inequality $g^{v}(N) \geq 0$ follows directly from the nonemptiness of the core of any convex game.

Next notice that for any $S \subseteq N$,

$$
g^{v}(N)-g^{v}(S)=\sum_{i \in N \backslash S}[v(N)-v(N \backslash\{i\})]-[v(N)-v(S)] .
$$

Denote elements of $N \backslash S$ by $i_{1}, i_{2}, \ldots i_{n-s}$, i.e., $N \backslash S=\left\{i_{1}, i_{2}, \ldots i_{n-s}\right\}$. Then,

$$
\begin{aligned}
& v(N)-v(S)= \\
& \quad\left[v(N)-v\left(N \backslash\left\{i_{1}\right\}\right)\right]+\left[v\left(N \backslash\left\{i_{1}\right\}\right)-v\left(N \backslash\left\{i_{1}, i_{2}\right\}\right)\right]+\ldots+\left[v\left(S \cup\left\{i_{n-s}\right\}\right)-v(S)\right] .
\end{aligned}
$$

Therefore, applying successively $n-s$ times the inequality (1), we obtain that for all $S \subseteq N, g^{v}(N)-g^{v}(S) \geq 0$.

A game $v \in \mathcal{G}_{N}$ is 1-convex if

$$
0 \leq g^{v}(N) \leq g^{v}(S), \quad \text { for all } S \subseteq N, S \neq \emptyset .
$$

As it is shown in Driessen and Tijs [7] and Driessen [5], every 1-convex game has a nonempty core. In a 1-convex game $v$, for every efficient vector $x \in \mathbb{R}^{n}$, the inequalities $x_{i} \leq m_{i}^{v}$, for all $i \in N$, guarantee that $x \in C(v)$. In particular, the characterizing property of a 1-convex game is that the replacement of any single coordinate $m_{i}^{v}$ in the vector $m^{v}$ by the amount of $v(N)-m^{v}(N \backslash i)$ places the resultant vector $\bar{m}^{v}(i)=\left\{\bar{m}_{j}^{v}(i)\right\}_{j \in N}$, given by

$$
\bar{m}_{j}^{v}(i)=\left\{\begin{array}{cl}
v(N)-m^{v}(N \backslash i)=m_{i}^{v}-g^{v}(N), & j=i, \\
m_{j}^{v}, & j \neq i,
\end{array} \quad \text { for all } j \in N,\right.
$$

into the core $C(v)$. Moreover, in a 1-convex game the set of vectors $\left\{\bar{m}^{v}(i)\right\}_{i \in N}$ creates a set of extreme points of the core which in turn coincides with their convex hull, i.e., $C(v)=\operatorname{co}\left(\left\{\bar{m}^{v}(i)\right\}_{i \in N}\right)$. Besides, the nucleolus $\nu(v)$ occupies the central position in the core coinciding with the barycenter of the core vertices, and is given by the formula

$$
\nu_{i}(v)=m_{i}^{v}-\frac{g^{v}(N)}{n}, \quad \text { for all } i \in N .
$$

So, the nucleolus coincides with the equal allocation of nonseparable contribution the amount of $g^{v}(N)$ over the players, or in other terms, every player according to nucleolus gets its marginal contribution to the grand coalition minus an equal share in the gap $g^{v}(N)$ of the grand coalition. That presents a special advantage of the class of 1-convex games because the nucleolus, defined as a solution to a lexicographical optimization problem that in general is difficult to compute, for 1-convex games appears to be linear and thus simple to determine.

By definition of 1-convexity (2) and from Proposition 1 we easily obtain 
Proposition 2 A convex game $v \in \mathcal{G}_{N}$ is 1-convex, if and only if

$$
g^{v}(N)=g^{v}(S), \quad \text { for all } S \subseteq N, S \neq \emptyset .
$$

In the next section we study the so-called co-insurance game that appears to be closely related to the well-known bankruptcy game. For a bankruptcy problem $(E ; d)$ given by an estate $E \in \mathbb{R}_{+}$and a vector of claims $d \in \mathbb{R}_{+}^{n}$ assuming that the total claim of the creditors is greater than the remaining estate, i.e., $d(N)=\sum_{i \in N} d_{i}>E$, the corresponding bankruptcy game $v_{E ; d} \in \mathcal{G}_{N}$ is defined in Aumann and Maschler [2] by

$$
v_{E ; d}(S)=\max \{0, E-d(N \backslash S)\}, \quad \text { for all } S \subseteq N .
$$

To conclude this section recall a few extra definitions that will be used below.

A set of coalitions $\mathcal{B} \subset 2^{N} \backslash\{N\}$ is called a set of balanced coalitions, if positive numbers $\lambda_{S}, S \in \mathcal{B}$ exist such that

$$
\sum_{S \in \mathcal{B}: S \ni i} \lambda_{S}=1, \quad \text { for all } i \in N .
$$

A player $i$ is a veto-player in the game $v \in \mathcal{G}_{N}$, if $v(S)=0$, for every $S \subseteq N \backslash i$. A game $v \in \mathcal{G}_{N}$ is a veto-rich game if it has at least one veto-player.

For a game $v \in \mathcal{G}_{N}$, a coalition $S \subseteq N, S \neq \emptyset$, and an efficient payoff vector $x \in \mathbb{R}^{n}$, the Davis-Maschler reduced game with respect to $S$ and $x$ is the game $v_{S, x} \in \mathcal{G}_{S}$ defined in [3] by

$$
v_{S, x}(T)=\left\{\begin{array}{cl}
0, & T=\emptyset, \\
v(N)-x(N \backslash S), & T=S, \\
\max _{Q \subseteq N \backslash S}(v(T \cup Q)-x(Q)), & \text { otherwise, }
\end{array} \text { for all } T \subseteq S .\right.
$$

\section{Co-insurance game and its core}

Consider the problem in which a risk is evaluated too much heavy for a single insurance company, but it can be insured by the finite set $N$ of companies that share a given risk $\mathcal{R}$ and premium $\Pi$. First, it is assumed that every company $i \in N$ expresses the valuation of a random variable $\mathcal{R}$ through a real-valued nonnegative functional $H_{i}(\mathcal{R})$ such that $H_{i}(0)=0$, for all $i \in N$. For any nonempty subset $S \subseteq N$ of companies, let $\mathcal{A}(S)=\left\{X \in \mathbb{R}^{S} \mid \sum_{i \in S} X_{i}=\mathcal{R}\right\}$ represents the (non-empty) set of feasible decompositions of the given risk $\mathcal{R}$. Second, by hypothesis, it is supposed, for every $S \subseteq N, S \neq \emptyset$, that an optimal decomposition of the risk exists, so that $\min _{X \in \mathcal{A}(S)} \sum_{i \in S} H_{i}\left(X_{i}\right):=\mathcal{P}(S)$ is well-defined. Here the real-valued set function $\mathcal{P}$ can be seen as the evaluation of the optimal decomposition of the risk $\mathcal{R}$ by the companies in coalition $S$ as a whole.

To determine the evaluation function $\mathcal{P}$ may result in general not an easy task. However, under some reasonable assumptions borrowed from real-life applications it turns out that $\mathcal{P}$ can be easily computed for all coalitions. For instance, in case of constant quotas, when it is supposed that for each insurable risk $\mathcal{R}$, for every $S \subseteq N, S \neq \emptyset$, there exists the only one feasible decomposition $\left(\frac{q_{i}}{q(S)} \mathcal{R}\right)_{i \in S} \in \mathbb{R}^{S}$ specified by a priori given quotas $q_{i}>0, i \in N, \sum_{i \in N} q_{i}=1$, and moreover, for each insurable risk $\mathcal{R}$,

$$
H_{i}(\mathcal{R})=q_{i} H\left(\frac{\mathcal{R}}{q_{i}}\right), \quad \text { for all } i \in N,
$$


where $H$ is some a priori fixed convex function, the evaluation function $\mathcal{P}$ for every $S \subseteq N, S \neq \emptyset$, is given by

$$
\mathcal{P}(S)=\sum_{i \in S} H_{i}\left(\frac{q_{i}}{q(S)} \mathcal{R}\right)=q(S) H\left(\frac{\mathcal{R}}{q(S)}\right) .
$$

If insurance companies evaluate a risk $\mathcal{R}$ according to the variance principle, i.e.,

$$
H_{i}(\mathcal{R})=E(\mathcal{R})+a_{i} \operatorname{Var}(\mathcal{R}), \quad a_{i}>0, \quad \text { for all } i \in N,
$$

where $E(\mathcal{R})$ and $\operatorname{Var}(\mathcal{R})$ denote the expectation and variance of a random variable $\mathcal{R}$, then we are in case of constant quotas when the corresponding quotas may be obtained as $q_{i}=\frac{a(N)}{a_{i}}$, where $a(N)=\left(\sum_{i \in N} \frac{1}{a_{i}}\right)^{-1}$ (cf. Deprez and Gerber [4], Fragnelli and Marina [8]). Later on we do not discuss the construction of the evaluation function $\mathcal{P}$. The only important in what follows is that $\mathcal{P}$ is nonnegative and non-increasing, i.e., for all $\emptyset \neq S \subseteq T \subseteq N, 0 \leq \mathcal{P}(T) \leq \mathcal{P}(S)$.

For a given premium $\Pi$ and an evaluation function $\mathcal{P}: 2^{N} \rightarrow \mathbb{R}$, Fragnelli and Marina [8] define the associated co-insurance game $v_{\Pi, \mathcal{P}} \in \mathcal{G}_{N}$ as following

$$
v_{\Pi, \mathcal{P}}(S)=\left\{\begin{array}{cc}
\max \{0, \Pi-\mathcal{P}(S)\}, & S \subseteq N, S \neq \emptyset, \\
0, & S=\emptyset .
\end{array}\right.
$$

By definition, the co-insurance game $v_{\Pi, \mathcal{P}}$ is nonnegative and since $\mathcal{P}$ is non-increasing it easily follows that $v$ is monotonic, i.e., for all $S \subseteq T \subseteq N, 0 \leq v_{\Pi, \mathcal{P}}(S) \leq v_{\Pi, \mathcal{P}}(T)$.

Notice that the well-known bankruptcy game (4) presents an example of the co-insurance game (5). Indeed, if for each insurance company $i \in N$ there exists a fixed "claim" $d_{i} \geq 0$ such that $\mathcal{P}(S)=\sum_{i \in N \backslash S} d_{i}$, for all $S \subseteq N, S \neq \emptyset$, then the co-insurance game reduces to the bankruptcy game with the estate equal to the premium $\Pi$. This particular evaluation function $\mathcal{P}$ is nonnegative and nonincreasing, $\mathcal{P}(N)=0$.

In the framework of the co-insurance game, we consider the evaluation function $\mathcal{P}$ being fixed, while the premium $\Pi$ as a variable quantity varying from small up to sufficiently large amounts. In order to avoid trivial situations, let the premium $\Pi$ be large enough so that $\Pi>\mathcal{P}(N)$. The following results are already proved in [8]:

- If the premium $\Pi$ is small enough in that $\Pi \leq \max _{i \in N} \mathcal{P}(N \backslash\{i\})$, then the coinsurance game $v_{\Pi, \mathcal{P}}$ is balanced since the core $C\left(v_{\Pi, \mathcal{P}}\right)$ contains the efficient allocation $\xi=\left\{\xi_{i}\right\}_{i \in N}$, where $\xi_{i^{*}}=v_{\Pi, \mathcal{P}}(N)$ for some $i^{*} \in \arg \max _{i \in N} \mathcal{P}(N \backslash\{i\})$, and $\xi_{i}=0$ for all $i \neq i^{*}$.

- If $\Pi>\bar{\alpha}_{\mathcal{P}}=\sum_{i \in N}[\mathcal{P}(N \backslash\{i\})-\mathcal{P}(N)]+\mathcal{P}(N)$, then $C\left(v_{\Pi, \mathcal{P}}\right)=\emptyset$.

- For all $\Pi \leq \bar{\alpha}_{\mathcal{P}}$, under the hypothesis of reduced concavity of function $\mathcal{P}$ :

$\mathcal{P}(S)-\mathcal{P}(S \cup\{i\}) \geq \mathcal{P}(N \backslash\{i\})-\mathcal{P}(N), \quad$ for all $S \varsubsetneqq N$ and every $i \in N \backslash S$,

$$
C\left(v_{\Pi, \mathcal{P}}\right) \neq \emptyset \text {. }
$$

To ensure strictly positive worth $v_{\Pi, \mathcal{P}}(S)>0$ for every coalition $S \subseteq N, S \neq \emptyset$, we suppose that the premium $\Pi$ is strictly bounded from below by the critical number $\underline{\alpha}_{\mathcal{P}}=\max _{i \in N} \mathcal{P}(\{i\})$. For all $\Pi \geq \underline{\alpha}_{\mathcal{P}}$, we have

$$
m_{i}^{v_{\Pi, \mathcal{P}}}=v_{\Pi, \mathcal{P}}(N)-v_{\Pi, \mathcal{P}}(N \backslash\{i\})=\mathcal{P}(N \backslash\{i\})-\mathcal{P}(N), \quad \text { for all } i \in N,
$$


for any $S \subseteq N, S \neq \emptyset$,

$$
g^{v_{\Pi, \mathcal{P}}}(S)=\sum_{i \in S} m_{i}^{v_{\Pi, \mathcal{P}}}-v_{\Pi, \mathcal{P}}(S)=\sum_{i \in S}[\mathcal{P}(N \backslash\{i\})-\mathcal{P}(N)]+\mathcal{P}(S)-\Pi .
$$

In what follows we distinguish the two cases $\bar{\alpha}_{\mathcal{P}} \geq \underline{\alpha}_{\mathcal{P}}$ and $\bar{\alpha}_{\mathcal{P}}<\underline{\alpha}_{\mathcal{P}}$.

Notice that in the bankruptcy setting, $\bar{\alpha}_{\mathcal{P}}=\sum_{i \in N} d_{i}$ and $\underline{\alpha}_{\mathcal{P}}=\sum_{i \in N} d_{i}-$ $\min _{i \in N} d_{i}$, i.e., it always holds that $\underline{\alpha}_{\mathcal{P}} \leq \bar{\alpha}_{\mathcal{P}}$.

First consider the case $\bar{\alpha}_{\mathcal{P}} \geq \underline{\alpha}_{\mathcal{P}}$. It turns out that in this case the nonemptiness of the core $C\left(v_{\Pi, \mathcal{P}}\right)$ for $\Pi=\bar{\alpha}_{\mathcal{P}}$ is equivalent to 1-convexity of the co-insurance game $v_{\bar{\alpha}_{\mathcal{P}}, \mathcal{P}}$.

Theorem 1 Let $\bar{\alpha}_{\mathcal{P}} \geq \underline{\alpha}_{\mathcal{P}}$, then the following equivalences hold:

(i) the co-insurance game $v_{\bar{\alpha}_{\mathcal{P}}, \mathcal{P}}$ is balanced;

(ii) the core $C\left(v_{\bar{\alpha}_{\mathcal{P}}, \mathcal{P}}\right)$ is a singleton and coincides with the marginal worth vector $m^{v_{\bar{\alpha}_{\mathcal{P}}, \mathcal{P}}}$

(iii) the evaluation function $\mathcal{P}$ meets the so-called 1-concavity condition

$$
\mathcal{P}(S)-\mathcal{P}(N) \geq \sum_{i \in N \backslash S}[\mathcal{P}(N \backslash\{i\})-\mathcal{P}(N)], \quad \text { for all } S \subseteq N, S \neq \emptyset ;
$$

(iv) the co-insurance game $v_{\bar{\alpha}_{\mathcal{P}}, \mathcal{P}}$ is 1-convex.

Proof From (8) it follows that for all $\Pi \geq \underline{\alpha}_{\mathcal{P}}$,

$$
\bar{\alpha}_{\mathcal{P}}=\sum_{i \in N}[\mathcal{P}(N \backslash\{i\})-\mathcal{P}(N)]+\mathcal{P}(N)=g^{v_{\Pi, \mathcal{P}}}(N)+\Pi .
$$

By hypothesis $\bar{\alpha}_{\mathcal{P}} \geq \underline{\alpha}_{\mathcal{P}}$, therefore, applying the last equality to $\Pi=\bar{\alpha}_{\mathcal{P}}$, we obtain that

$$
g^{v_{\bar{\alpha}}, \mathcal{P}}(N)=0 .
$$

Since for any game $v \in \mathcal{G}_{N}$, the marginal worth vector $m^{v}$ provides upper bound for the core, a game $v$ with zero gap $g^{v}(N)=0$ can possess at most one core allocation coinciding with $m^{v}$, which is $m^{v_{\overline{\mathcal{A}}_{\mathcal{P}}, \mathcal{P}}}$ in case of the co-insurance game $v_{\bar{\alpha}_{\mathcal{P}}, \mathcal{P}}$. Next notice that the 1-concavity condition (9) is equivalent to

$\sum_{i \in S}[\mathcal{P}(N \backslash\{i\})-\mathcal{P}(N)] \geq \sum_{i \in N}[\mathcal{P}(N \backslash\{i\})-\mathcal{P}(N)]+\mathcal{P}(N)-\mathcal{P}(S)$, for all $S \subseteq N, S \neq \emptyset$,

which is the same as the marginal worth vector $m^{v_{\bar{\alpha}_{\mathcal{P}}, \mathcal{P}}}$ satisfies the core constraints

$$
\sum_{i \in S} m_{i}^{v_{\bar{\alpha}_{\mathcal{P}}, \mathcal{P}}} \geq \bar{\alpha}_{\mathcal{P}}-\mathcal{P}(S)=v_{\bar{\alpha}_{\mathcal{P}}, \mathcal{P}}(S), \quad \text { for all } S \subseteq N, S \neq \emptyset .
$$

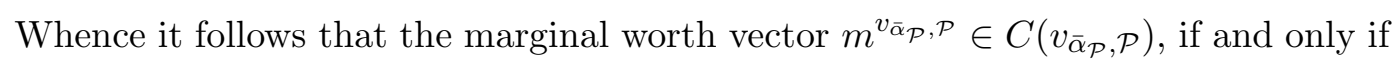
the evaluation function $\mathcal{P}$ satisfies the 1-concavity condition (9). Moreover, because of (8), the inequality (11) is equivalent to

$$
g^{v_{\bar{\alpha}_{\mathcal{P}}, \mathcal{P}}}(N) \leq g^{v_{\bar{\alpha}_{\mathcal{P}}, \mathcal{P}}}(S), \quad \text { for all } S \subseteq N, S \neq \emptyset,
$$

which together with equality (10) is equivalent to 1-convexity of the co-insurance game $v_{\bar{\alpha}_{\mathcal{P}}, \mathcal{P}}$. 
Remark 1 Notice that our 1-concavity condition (9) is weaker then the condition of reduced concavity (6) used in [8].

Theorem 2 If for some fixed premium $\Pi^{*} \geq \underline{\alpha}_{\mathcal{P}}$, the co-insurance game $v_{\Pi^{*}, \mathcal{P}}$ is 1-convex, then for every premium $\Pi, \underline{\alpha}_{\mathcal{P}} \leq \Pi \leq \Pi^{*}$, the corresponding co-insurance game $v_{\Pi, \mathcal{P}}$ is 1-convex as well.

Proof For all $\Pi \geq \underline{\alpha}_{\mathcal{P}}$, due to (8) it holds that for every $S \subseteq N, S \neq \emptyset$, the gap $g^{v_{\Pi, \mathcal{P}}}(S)$ is a decreasing linear function of the variable $\Pi$, while the difference $g^{v_{\Pi, \mathcal{P}}}(S)-g^{v_{\Pi, \mathcal{P}}}(N)$ is constant for all $\Pi$. Whence, it follows that if for some fixed premium $\Pi^{*} \geq \underline{\alpha}_{\mathcal{P}}$ the co-insurance game $v_{\Pi^{*}, \mathcal{P}}$ is 1-convex, i.e., for all $S \subseteq N$, $S \neq \emptyset$, the inequality (2) holds, then this inequality remains valid for all premium $\underline{\alpha}_{\mathcal{P}} \leq \Pi \leq \Pi^{*}$, i.e., all games $v_{\Pi, \mathcal{P}}$ appear to be 1-convex as well.

The next theorem follows easily from Theorem 1 and Theorem 2 .

Theorem 3 Let $\bar{\alpha}_{\mathcal{P}} \geq \underline{\alpha}_{\mathcal{P}}$. If the evaluation function $\mathcal{P}$ satisfies the 1-concavity condition (9), then for any premium $\underline{\alpha}_{\mathcal{P}} \leq \Pi \leq \bar{\alpha}_{\mathcal{P}}$,

(i) the corresponding co-insurance game $v_{\Pi, \mathcal{P}}$ is 1-convex;

(ii) the core $C\left(v_{\Pi, \mathcal{P}}\right) \neq \emptyset$;

(iii) the nucleolus $\nu\left(v_{\Pi, \mathcal{P}}\right)$ is the barycenter of the core $C\left(v_{\Pi, \mathcal{P}}\right)$ and is given by

$$
\nu_{i}\left(v_{\Pi, \mathcal{P}}\right)=\mathcal{P}(N \backslash\{i\})-\mathcal{P}(N)+\frac{\Pi-\bar{\alpha}_{\mathcal{P}}}{n}, \quad \text { for all } i \in N .
$$

Proof The first statement follows directly from Theorem 1 and Theorem 2. Next, recall already mentioned above results obtained in Driessen and Tijs [7] and Driessen [5], stating that every 1-convex game has a nonempty core and its nucleolus being the barycenter of the core is given by the formula (3). These facts, together with (7) and (8), complete the proof.

In words, the third statement of Theorem 3 means that the nucleolus of these co-insurance games is a linear function of the variable premium such that each incremental premium is shared equally among the insurance companies. Geometrically, the nucleoli payoffs follow a straight line to end up at the marginal worth vector yielding payoff $\mathcal{P}(N \backslash\{i\})-\mathcal{P}(N)$ to player $i \in N$.

Remark 2 The statement of Theorem 3 remains in force if the 1-concavity condition (9) for the evaluation function $\mathcal{P}$ is replaced by any one of the equivalent conditions given by Theorem 1, in particular if $C\left(v_{\bar{\alpha}_{\mathcal{P}}, \mathcal{P}}\right) \neq \emptyset$ or if the co-insurance game $v_{\bar{\alpha}_{\mathcal{P}}, \mathcal{P}}$ is 1-convex.

Remark 3 Formula (12) for nucleolus of a co-insurance game can be derived alternatively using the method for computing the nucleolus of the so-called compromise stable game introduced in Quant et al. [11]. Indeed, it is not difficult to check that every 1-convex game appears to be compromise stable. 
Remark 4 In the bankruptcy setting Theorem 3 expresses the fact that the nucleolus provides equal losses to all creditors (insurance companies) with respect to their individual claims, if estate (premium) varies between $\sum_{i \in N} d_{i}-\min _{i \in N} d_{i}$ and $\sum_{i \in N} d_{i}$, which agrees well with the Talmud rule for bankruptcy situations studied exhaustively in Aumann and Maschler [2].

Consider now the case $\bar{\alpha}_{\mathcal{P}}<\underline{\alpha}_{\mathcal{P}}$. In this case, even if the co-insurance game $v_{\bar{\alpha}_{\mathcal{P}}, \mathcal{P}}$ is 1-convex, for the co-insurance game $v_{\Pi, \mathcal{P}}$ corresponding to the premium $\Pi<\bar{\alpha}_{\mathcal{P}}$ the 1 -convexity may be lost immediately while lowering the premium. This happens due to the fact that the co-insurance worth of at least one coalition turns out to be at zero level. For instance, consider the following example.

Example 1 Let the evaluation function $\mathcal{P}$ for 3 insurance companies be given by $\mathcal{P}(\{1\})=5, \mathcal{P}(\{2\})=4, \mathcal{P}(\{3\})=3, \mathcal{P}(\{1,2\})=\mathcal{P}(\{1,3\})=\mathcal{P}(\{2,3\})=2$, and $\mathcal{P}(\{1,2,3\})=1$. In this case, $4=\bar{\alpha}_{\mathcal{P}}<\underline{\alpha}_{\mathcal{P}}=5$.

- If the premium $\Pi=4$, then the co-insurance game $v_{4, \mathcal{P}}$ :

$v_{4, \mathcal{P}}(\{1\})=v_{4, \mathcal{P}}(\{2\})=0, v_{4, \mathcal{P}}(\{3\})=1, v_{4, \mathcal{P}}(\{12\})=v_{4, \mathcal{P}}(\{13\})=v_{4, \mathcal{P}}(\{23\})=$ $2, v_{4, \mathcal{P}}(\{123\})=3$,

is a 1-convex game with the minimal for a 1-convex game gap $g^{v_{4, \mathcal{P}}}(\{123\})=0$ and, therefore, with the unique core allocation $m^{v_{4, \mathcal{P}}}=(1,1,1)$.

- If the premium $\Pi=3$, then the co-insurance game $v_{3, \mathcal{P}}$ :

$v_{3, \mathcal{P}}(\{1\})=v_{3, \mathcal{P}}(\{2\})=v_{3, \mathcal{P}}(\{3\})=0, v_{3, \mathcal{P}}(\{12\})=v_{3, \mathcal{P}}(\{13\})=v_{3, \mathcal{P}}(\{23\})=1$, $v_{3, \mathcal{P}}(\{123\})=2$,

is a symmetric 1-convex and convex, since the gap $g^{v_{3, \mathcal{P}}}(S)=1$ is constant for all $S \subseteq N, S \neq \emptyset$, while its core $C\left(v_{3, \mathcal{P}}\right)$ is the triangle with three extreme points $(1,1,0),(1,0,1),(0,1,1)$.

- For any premium $2 \leq \Pi<3$, the corresponding co-insurance game $v_{\Pi, \mathcal{P}}$ is zeronormalized and symmetric: $v_{\Pi, \mathcal{P}}(i)=0, v_{\Pi, \mathcal{P}}(i j)=\Pi-2, \quad v_{\Pi, \mathcal{P}}(123)=\Pi-1$.

However, the 1-convexity fails because the gap of singletons is strictly less than the gap of $N: g^{v_{\Pi, \mathcal{P}}}(i)=1<4-\Pi=g^{v_{\Pi, \mathcal{P}}}(123)$.

\section{Algorithms for computing nucleolus}

It is easy to compute the nucleolus of a co-insurance game when it is a linear function of a given premium as it is stated by Theorem 3. In this section we introduce a comparatively simple algorithm that allows to compute the nucleolus of a co-insurance game also in cases when it is nonlinear in the premium. To do that, we uncover first the relation between the class of co-insurance games, in particular bankruptcy games, and the class Davis-Maschler reduced games of monotonic vetorich games obtained by deleting a veto-player with respect to the nucleolus. Second, we provide an algorithm for computing the nucleolus for games of the latter class.

In what follows by $\mathcal{G}_{N}^{m}$ we denote the class of all monotonic games with a player set $N$. Let $N_{0}:=N \cup\{0\}$ and $n_{0}=n+1$. Consider the class $\mathcal{G}_{N_{0}}^{m}$ of monotonic veto-rich games with a player set $N_{0}$ and the player 0 being a veto-player. Besides, 
we consider the class $\mathcal{G}_{N_{0}}^{+}$of nonnegative veto-rich games with a player set $N_{0}$ and the player 0 being a veto-player, that satisfies also the property $v^{0}\left(N_{0}\right) \geq v^{0}(S)$, for all $S \subseteq N_{0}$. It is easy to see that $\mathcal{G}_{N_{0}}^{m} \subset \mathcal{G}_{N_{0}}^{+}$. Define $\mathcal{R}_{N}$ as a class of veto-removed games $v \in \mathcal{G}_{N}$ that are the Davis-Maschler reduced games of games $v^{0} \in \mathcal{G}_{N_{0}}^{m}$ obtained by deleting the veto-player 0 in accordance to the nucleolus payoff. As it was already shown in Arin and Feltkamp [1], for every veto-rich game from the class $\mathcal{G}_{N_{0}}^{+}$the core is nonempty and the nucleolus payoff to a veto-player is larger than or equal to that of the other players. From where it easily follows that every veto-removed game is balanced because the Davis-Maschler reduced game inherits the core property and, moreover, in every nontrivial veto-rich game $v^{0} \in \mathcal{G}_{N_{0}}^{+}$the nucleolus payoff to a veto-player $\nu_{0}\left(v^{0}\right)>0$ since in any nontrivial game $v^{0} \in \mathcal{G}_{N_{0}}^{+}$ the worth of the grand coalition $v^{0}\left(N_{0}\right)>0$.

Some extra notation. With every game $v \in \mathcal{G}_{N}^{+}$we associate the following vetorich game $v^{0} \in \mathcal{G}_{N_{0}}^{+}$defined as

$$
v^{0}(S)=\left\{\begin{array}{cl}
0, & S \not \supset 0, \\
v(S \backslash\{0\}), & S \ni 0,
\end{array} \quad \text { for all } S \subseteq N_{0} .\right.
$$

For every veto-rich game $v^{0} \in \mathcal{G}_{N_{0}}^{+}$let $\nu_{0}$ denote the nucleolus payoff $\nu_{0}\left(v^{0}\right)$ to the veto-player 0 in $v^{0}$. Besides, for a game $v \in \mathcal{G}_{N}$ and $a \in \mathbb{R}_{+}$we define the game $v^{-a} \in \mathcal{G}_{N}$ as follows

$$
v^{-a}(S)=\max \{0, v(S)-a\}, \quad \text { for all } S \subseteq N .
$$

Below for the facilitation of reading for any set of players $M$ containing the vetoplayer 0 , any coalition $S_{0} \subseteq M$ with subindex 0 is assumed to contain the veto-player 0 , and it is supposed that for any $S_{0} \subseteq M$ holds the equality $s_{0}=\left|S_{0}\right|=s+1$, where $s=\left|S_{0} \cap M \backslash\{0\}\right|$. Furthermore, for any game $w \in \mathcal{G}_{M}$, for every $S \varsubsetneqq M$ we define a number

$$
\kappa_{w}(S)=\left\{\begin{array}{cl}
\frac{w(M)-w(S)}{m-s+1}, & S \neq \emptyset, \\
\frac{w(M)}{m}, & S=\emptyset .
\end{array}\right.
$$

For $M \not \supset 0$, we define also a number $\kappa^{*}(w)=\min _{S \varsubsetneqq M} \kappa_{w}(S)$, and for $M \ni 0$, we define a number $\kappa_{0}^{*}(w)=\min _{S_{0} \varsubsetneqq M} \kappa_{w}\left(S_{0}\right)$.

Theorem 4 It holds that

(i) every game $v \in \mathcal{R}_{N}$ can be presented in the form of a co-insurance game $v_{\Pi, \mathcal{P}} \in \mathcal{G}_{N} ;$

(ii) if $v_{\Pi^{*}, \mathcal{P}} \in \mathcal{R}_{N}$, then for every premium $\Pi \leq \Pi^{*}, v_{\Pi, \mathcal{P}} \in \mathcal{R}_{N}$ as well;

(iii) for every evaluation function $\mathcal{P}: 2^{N} \rightarrow \mathbb{R}$, for every premium $\Pi$,

$$
\Pi \leq \Pi^{*}=\mathcal{P}(N)+n \min _{S \subsetneq N} \frac{\mathcal{P}(S)-\mathcal{P}(N)}{n-s+1},
$$

the co-insurance game $v_{\Pi, \mathcal{P}} \in \mathcal{R}_{N}$. 
Proof $(i)$. Consider $v \in \mathcal{R}_{N}$. By definition of $\mathcal{R}_{\mathcal{N}}$ there exists $v^{0} \in \mathcal{G}_{N_{0}}^{m}$ such that $v$ is the Davis-Maschler reduced game derived from $v^{0}$ by deleting the veto-player 0 with respect to the nucleolus. By definition of the Davis-Maschler reduced game it holds

$$
v(S)=\left\{\begin{array}{cc}
0, & S=\emptyset, \\
v^{0}(N \cup\{0\})-\nu_{0}, & S=N, \\
\max \left\{v^{0}(S), v^{0}(S \cup\{0\})-\nu_{0}\right\}=\max \left\{0, v^{0}(S \cup\{0\})-\nu_{0}\right\}, & S \varsubsetneqq N, S \neq \emptyset .
\end{array}\right.
$$

Take some positive $k>\frac{v^{0}(N \cup\{0\})}{\nu_{0}}-1$ and set

$$
\begin{gathered}
\Pi=k \nu_{0}, \\
\mathcal{P}(S)=(k+1) \nu_{0}-v^{0}(S \cup\{0\}), \quad \text { for all } S \subseteq N, S \neq \emptyset .
\end{gathered}
$$

Whence

$$
v(S)=\max \{0, \Pi-\mathcal{P}(S)\}, \quad \text { for all } S \subseteq N, S \neq \emptyset .
$$

(ii). Recall first that every co-insurance game is monotonic and, moreover, for any co-insurance game $v_{\Pi, \mathcal{P}}$, for any $a \in \mathbb{R}_{+}$, the game $v_{\Pi, \mathcal{P}}^{-a}$ is also a co-insurance game with the premium equal to $\Pi-a$, i.e, $v_{\Pi, \mathcal{P}}^{-a}=v_{\Pi-a, \mathcal{P}}$. Therefore in view of $(i)$ proved above, for proving $(i i)$ it is sufficient to show that if for certain game $v \in \mathcal{G}_{N}^{m}$ it holds that $v^{-a} \in \mathcal{R}_{N}$ for some $a \in \mathbb{R}_{+}$, then $v^{-b} \in \mathcal{R}_{N}$ for all $b \in \mathbb{R}_{+}, a<b$. Moreover, notice that it is enough to prove that $v^{-b} \in \mathcal{R}_{N}$ only for $a<b \leq v(N)$ since due to (14), it holds $v^{-b} \equiv 0 \in \mathcal{R}_{N}$ for all $b \geq v(N)$.

Consider now a game $v \in \mathcal{G}_{N}^{m}$ together with its associated veto-rich game $v^{0} \in$ $\mathcal{G}_{N_{0}}^{m}$. From (13) and already mentioned above statement of Arin and Feltkamp [1] concerning the nucleolus payoff to a veto-player, it follows easily that $\frac{v(N)}{n+1} \leq \nu_{0} \leq$ $v(N)$. Set $a:=\nu_{0}$. It is not difficult to see that $v^{-a} \in \mathcal{G}_{N}$ is the Davis-Maschler reduced game of the game $v^{0}$ obtained by deleting the veto-player 0 with respect to the nucleolus payoff $a$. So, by definition of $\mathcal{R}_{N}, v^{-a} \in \mathcal{R}_{N}$. Recall that if $a=v(N)$, $v^{-a} \equiv 0 \in \mathcal{R}_{N}$.

Next, we show that if $a<v(N)$, then for all $b, a \leq b \leq v(N)$, also $v^{-b} \in \mathcal{R}_{N}$. The above procedure of constructing a veto-removed game may be applied to any monotonic game, in particular to the just obtained monotonic game $v^{-a} \in \mathcal{R}_{N}$. Doing that, we get another monotonic game, say $v^{-a^{1}} \in \mathcal{R}_{N}$, with $a^{1}=a+\nu_{0}\left(v^{-a}\right)>$ $a$ when $a<v(N)$. We show first that $v^{-b} \in \mathcal{R}_{N}$ for all $a \leq b \leq a^{1}$. Consider $0 \leq c \leq a$ and apply the above procedure for all monotonic games $v^{-c} \in \mathcal{G}_{N}$. For $c=0$ we start with $v$ and obtain the monotonic game $v^{-a} \in \mathcal{R}_{N}$. For $c=a$ we start with $v^{-a}$ and obtain the monotonic game $v^{-a^{1}} \in \mathcal{R}_{N}$. Due to the continuity of the nucleolus we obtain all $v^{-b}$ while $c$ varies between 0 and $a$. Hence $v^{-b} \in \mathcal{R}_{N}$ for all $a \leq b \leq a^{1}$.

When $a^{1}<v(N)$ then applying the above procedure to the game $v^{-a^{1}}$ we obtain a game $v^{-a^{2}} \in \mathcal{R}_{N}$ with $a^{2}>a^{1}$ and so on. Since on each step $k, a^{k}-a^{k-1}=$ $\nu_{0}\left(v^{-a^{k-1}}\right) \geq \frac{v(N)-a^{k-1}}{n+1}$, any number $a \leq b \leq v(N)$ can be reached by not more than $\frac{b-a}{v(N)-b}(n+1)$ steps. Therefore, for every $b, a \leq b \leq v(N), v^{-b} \in \mathcal{R}_{N}$.

(iii). Take a co-insurance game $v_{\Pi, \mathcal{P}}$ with $\Pi \geq \underline{\alpha}_{\mathcal{P}}$, for simplicity of notation denote $v_{\Pi, \mathcal{P}}$ by $v$, and consider the corresponding veto-rich game $v^{0} \in \mathcal{G}_{N_{0}}^{m}$ defined by (13). As it is shown in the proof of (ii), the Davis-Maschler reduced game of 
the game $v^{0}$ obtained by deleting the veto-player 0 with respect to the nucleolus coincides with the game $v^{-a}, a=\nu_{0}$, which in turn coincides with the co-insurance game $v_{\Pi^{\prime}, \mathcal{P}}$ with $\Pi^{\prime}=\Pi-\nu_{0}$. Hence, $v_{\Pi^{\prime}, \mathcal{P}} \in \mathcal{R}_{N}$. From Proposition 3 below and the definition of a co-insurance game (5), since $\Pi \geq \underline{\alpha}_{\mathcal{P}}$, it follows that

$$
\nu_{0} \leq \Pi-\mathcal{P}(N)-n \min _{S \varsubsetneqq N} \frac{\mathcal{P}(S)-\mathcal{P}(N)}{n-s+1},
$$

i.e.,

$$
\Pi^{\prime} \geq \mathcal{P}(N)+n \min _{S \varsubsetneqq N} \frac{\mathcal{P}(S)-\mathcal{P}(N)}{n-s+1} .
$$

Then the validity of $(i i i)$ follows immediately from the just proved $(i i)$.

Notice that (16) provides rather rough estimation of $\Pi^{*}$. In fact, in the particular case of bankruptcy games, (16) guarantees that $v_{E ; d} \in \mathcal{R}_{N}$ only when $E \leq 0$. Next theorem imposes weaker conditions on the parameters of a bankruptcy game $v_{E ; d}$ to guarantee that $v_{E ; d} \in \mathcal{R}_{N}$.

Theorem 5 For any estate $E \in \mathbb{R}_{+}$and any vector of claims $d \in \mathbb{R}_{+}^{n}$ such that

$$
E \leq \frac{\sum_{i=1}^{n} d_{i}}{2}
$$

the corresponding bankruptcy game $v_{E ; d} \in \mathcal{R}_{N}$.

Proof First take a bankruptcy game $v_{E ; d}$ with $E=\sum_{i \in N} d_{i}$ and let $v$ be its coinsurance game representation, i.e., $v=v_{\Pi, \mathcal{P}}$ with $\Pi=d(N)$ and $\mathcal{P}(S)=d(N \backslash S)$. For a co-insurance game $v$ consider the corresponding veto-rich game $v^{0}$ defined by $(13)$,

$$
v^{0}(S)=\left\{\begin{array}{cl}
0, & S \not \supset 0, \\
\sum_{i \in S \backslash\{0\}} d_{i}, & S \ni 0,
\end{array} \quad \text { for all } S \subseteq N_{0} .\right.
$$

We compute now the nucleolus payoff $\nu_{0}$ to the veto player 0 in $v^{0}$ applying Algorithm 2 yielding nucleolus for monotonic veto-rich games with a veto-player 0 introduced below. Without loss of generality we assume that $d_{1} \leq d_{2} \leq \ldots \leq d_{n}$. Moreover, for every $k=1, \ldots, n$ we define a veto-rich game $v^{k}$ on $N_{0} \backslash\{1, \ldots, k\}$ as follows

$$
v^{k}(S)=\left\{\begin{array}{ll}
0, & S \not \supset 0, \\
\sum_{i \in S \backslash\{0\}} d_{i}+\sum_{i=1}^{k} \frac{d_{i}}{2}, & S \ni 0,
\end{array} \quad \text { for all } S \subseteq N_{0} .\right.
$$

For any coalition $S_{0} \varsubsetneqq N_{0}$ it holds that

$$
\kappa_{v^{0}}\left(S_{0}\right)=\frac{v^{0}\left(N_{0}\right)-v^{0}\left(S_{0}\right)}{n-s+1}=\frac{d\left(N_{0} \backslash S_{0}\right)}{n-s+1} \geq \frac{d_{1}(n-s)}{n-s+1} \geq \frac{d_{1}}{2}=\kappa_{v^{0}}\left(N_{0} \backslash\{1\}\right) .
$$

Whence it follows that $\kappa_{0}^{*}\left(v^{0}\right)=\kappa_{v^{0}}\left(N_{0} \backslash\{1\}\right)$, and therefore, Step 1 of Algorithm 2 assigns the nucleolus payoff $\nu_{1}\left(v^{0}\right)=\frac{d_{1}}{2}$ to the player 1. Moreover, the DavisMaschler reduced game constructed in Step 1 is defined on the player set $N_{0} \backslash\{1\}$ 
and coincides with the game $v^{1}$. Using the similar reasoning it is not difficult to see that for any $k=2, \ldots, n$, Algorithm 2 applied to the veto-rich game $v^{k-1}$ defined on the player set $N_{0} \backslash\{1, \ldots, k-1\}$ assigns the nucleolus payoff $\frac{d_{k}}{2}$ to the player $k$ and goes to the next step with the Davis-Maschler reduced game coinciding with the game $v^{k}$ defined on the player set $N_{0} \backslash\{1, \ldots, k\}$. Then applying the induction argument we obtain that $\nu_{i}\left(v^{0}\right)=\frac{d_{i}}{2}$ for all $i \in N$ and $\nu_{0}=\nu_{0}\left(v^{0}\right)=\frac{d(N)}{2}$.

Next observe that if a co-insurance game $v_{\Pi, \mathcal{P}}$ represents a bankruptcy game $v_{E ; d}$, then for any $a \in \mathbb{R}_{+}$, the co-insurance game $v_{\Pi, \mathcal{P}}^{-a}$ represents the bankruptcy game $v_{E-a ; d}$. Hence, we may complete the proof following the same arguments as in the proof of the statement (ii) of Theorem 4.

Consider now the following algorithm for constructing a payoff vector, say $x \in$ $\mathbb{R}^{N}$, in a game $v \in \mathcal{R}_{N}$.

\section{Algorithm 1}

0. Set $M=N$ and $w=v$.

1. Find a coalition $S \varsubsetneqq M$ with minimal size such that $\kappa_{w}(S)=\kappa^{*}(w)$.

2. For $i \in M \backslash S$, set $x_{i}=\kappa_{w}(S)$. If $S=\emptyset$, then stop, otherwise go to Step 3.

3. Construct the Davis-Maschler reduced game $w_{S, x} \in \mathcal{G}_{S}$. Set $M=S$ and $w=w_{S, x}$ and return to Step 1.

Theorem 6 For any veto-removed game $v \in \mathcal{R}_{N}$, Algorithm 1 yields the nucleolus payoff, i.e., $x=\nu(v)$.

The proof of Theorem 6 is obtained by comparing the outputs of two algorithms yielding nucleoli - Algorithm 1 applied to a veto-removed game $v \in \mathcal{R}_{N}$ and another Algorithm 2, applied to the associated monotonic veto-rich game $v^{0} \in \mathcal{G}_{N_{0}}^{m}$. Algorithm 2 is closed conceptually to the algorithm for computing the nucleolus for veto-rich games suggested in Arin and Feltkamp [1]. It is worth noting that for the application of Algorithm 1 to a veto-removed game $v \in \mathcal{R}_{N}$ there is no need in construction of the associated monotonic veto-rich game $v^{0} \in \mathcal{G}_{N_{0}}^{m}$ which is only necessary for proving Theorem 6 . The proof of Theorem 6 is given after the proof of Theorem 7 .

The following Algorithm 2 constructs a payoff vector, say $y \in \mathbb{R}^{N_{0}}$, in a game $v^{0} \in \mathcal{G}_{N_{0}}^{+}$. Since $\mathcal{G}_{N_{0}}^{m} \subset \mathcal{G}_{N_{0}}^{+}$, Algorithm 2 is applicable to any game $v^{0} \in \mathcal{G}_{N_{0}}^{m}$ as well.

\section{Algorithm 2}

0. Set $M=N_{0}$ and $w=v^{0}$.

1. Find a coalition $S_{0} \varsubsetneqq M$ with minimal size such that $\kappa_{w}\left(S_{0}\right)=\kappa_{0}^{*}(w)$.

2. For $i \in M \backslash S_{0}$, set $y_{i}=\kappa_{w}\left(S_{0}\right)$. If $S_{0}=\{0\}$, set $y_{0}=v^{0}\left(N_{0}\right)-\sum_{i \in N} y_{i}$ and stop, otherwise go to Step 3.

3. Construct the Davis-Maschler reduced game $w_{S_{0}, y} \in \mathcal{G}_{S_{0}}$. Set $M=S_{0}$ and $w=w_{S_{0}, y}$ and return to Step 1 . 
Theorem 7 For any veto-rich game $v^{0} \in \mathcal{G}_{N_{0}}^{+}$, Algorithm 2 yields the nucleolus payoff, i.e., $y=\nu\left(v^{0}\right)$.

Proof Let $v^{0} \in \mathcal{G}_{N_{0}}^{+}$. For the simplification of notation denote the nucleolus $\nu\left(v^{0}\right)$ by $x, x \in \mathbb{R}^{n+1}$, and let $e^{*}\left(v^{0}\right)$ denote the maximal excess with respect to the nucleolus in the game $v^{0}$, i.e., $e^{*}\left(v^{0}\right)=\max _{S \subsetneq N_{0}} e^{v^{0}}(S, x)$. As a corollary to the Kohlberg's characterization of the prenucleolus [10] it holds that the collection of coalitions with maximal excess values with respect to the nucleolus is balanced. Due to the balancedness, among the coalitions having the maximal excess there exists $S_{0} \varsubsetneqq N_{0}$. We show that every singleton $\{i\}, i \notin S_{0}$, also has the maximal excess. Let $i \notin S_{0}$. Again due to the balancedness, there exists $S \subset N_{0}, S \ni i, S \not \ngtr 0$, with maximal excess. Observe that since $S \not \supset 0$, then by definition of a veto-rich game $v^{0}(S)=v^{0}(S \backslash\{i\})=v^{0}(\{i\})=0$. If $|S|>1$ then

$$
e(S, x)=v^{0}(S)-x(S)=-x(S)=-x(\{i\})-x(S \backslash\{i\})=e(\{i\}, x)+e(S \backslash\{i\}, x) .
$$

Since the core of every veto-rich game in $\mathcal{G}_{+}^{N_{0}}$ is nonempty, the nucleolus belongs to the core and all excesses with respect to the nucleolus are nonpositive, in particular, $e(S \backslash\{i\}, x) \leq 0$. From where it follows that $e(\{i\}, x) \geq e(S, x)$, i.e., every singleton $\{i\}, i \notin S$, possesses the maximal excess as well.

For every $S_{0} \varsubsetneqq N_{0}$ with maximal excess with respect to the nucleolus from the efficiency of the nucleolus and the equality $v^{0}(\{i\})=0$ for all $i \in N_{0} \backslash S_{0}$, it follows that

$$
\begin{aligned}
& v^{0}\left(S_{0}\right)-v^{0}\left(N_{0}\right)=v^{0}\left(S_{0}\right)-x\left(N_{0}\right)=v^{0}\left(S_{0}\right)-x\left(S_{0}\right)-\sum_{i \in N_{0} \backslash S_{0}} x(i)= \\
&=e^{v^{0}}\left(S_{0}, x\right)+\sum_{i \in N_{0} \backslash S_{0}} e^{v^{0}}(\{i\}, x)=e^{*}\left(v^{0}\right) \cdot\left(n_{0}-s_{0}+1\right)=e^{*}\left(v^{0}\right) \cdot(n-s+1) .
\end{aligned}
$$

Moreover, for every $T_{0} \varsubsetneqq N_{0}$ it holds that

$$
v^{0}\left(T_{0}\right)-v^{0}\left(N_{0}\right)=e^{v^{0}}\left(T_{0}, x\right)+\sum_{i \in N_{0} \backslash T_{0}} e^{v^{0}}(\{i\}, x) \leq e^{*}\left(v^{0}\right) \cdot(n-t+1) .
$$

Whence, for every $T_{0} \varsubsetneqq N_{0}$

$$
\kappa_{v^{0}}\left(T_{0}\right) \stackrel{(15)}{=}-\frac{v^{0}\left(T_{0}\right)-v^{0}\left(N_{0}\right)}{n_{0}-t_{0}+1} \geq-e^{*}\left(v^{0}\right)
$$

while for $S_{0} \varsubsetneqq N_{0}$ with maximal excess with respect to the nucleolus holds the equality $\kappa_{v^{0}}\left(S_{0}\right)=-e^{*}\left(v^{0}\right)$. Then it follows that $S_{0} \varsubsetneqq N_{0}$ has the maximal excess with respect to the nucleolus if and only if $\kappa_{v^{0}}\left(S_{0}\right)=\kappa_{0}^{*}\left(v^{0}\right)$.

Therefore, on the first iteration of Algorithm 2 when $M=N_{0}$ and $w=v^{0}$, Step 1 provides a coalition $S_{0} \varsubsetneqq N_{0}$ with maximal excess with respect to the nucleolus. Then Step 2 assigns to every $i \in N_{0} \backslash S_{0}$ its nucleolus payoff because the assigned payoff $y_{i}=\kappa_{v^{0}}\left(S_{0}\right)$ coincides with $x_{i}=\nu_{i}\left(v^{0}\right)$ since

$$
y_{i}=\kappa_{v^{0}}\left(S_{0}\right)=-e^{*}\left(v^{0}\right)=-\left(v^{0}(\{i\})-x_{i}\right)=x_{i}, \quad \text { for all } i \in N_{0} \backslash S_{0} .
$$


In every veto-rich game from the class $\mathcal{G}_{M}^{+}$with $M$ containing the veto-player 0 the nucleolus coincides with the prenucleolus due to the nonemptiness of the core which was already mentioned above with reference to [1]. Then, because of the Davis-Maschler consistency of the prenucleolus [14], the nucleolus payoffs to the players in the Davis-Maschler reduced game $w_{S_{0}, y} \in \mathcal{G}_{S_{0}}$ constructed in Step 3 of Algorithm 2 are the same as the nucleolus payoffs to the players in $S_{0}$ in the game $w \in \mathcal{G}_{M}^{+}$. Thus in order to complete the proof, it only remains to show that the Davis-Maschler reduced game $w_{S_{0}, y} \in \mathcal{G}_{S_{0}}$ of a game $w \in \mathcal{G}_{+}^{M}$ with $M$ containing the veto-player 0 is itself a veto-rich game belonging to $\mathcal{G}_{S_{0}}^{+}$.

Take $T \subseteq S_{0} \backslash\{0\}$. Then

$$
w_{S_{0}, y}(T)=\max _{Q \subseteq M \backslash S_{0}}(w(T \cup Q)-y(Q))=\max _{Q \subseteq M \backslash S_{0}}\{0-y(Q)\}=0,
$$

because $w(T \cup Q)=0$ for every $w \in \mathcal{G}_{M}^{+}$, since $T \cup Q \subseteq M \backslash\{0\}$ for every $Q \subseteq M \backslash S_{0}$. Thus, 0 is a veto-player in $w_{S_{0}, y} \in \mathcal{G}_{S_{0}}$ as well. Further, it is evident that $w_{S_{0}, y}$ is nonnegative. Hence, it remains to show that $w_{S_{0}, y}\left(S_{0}\right) \geq w_{S_{0}, y}(T)$ for every $T \subseteq S_{0}$. When $T \subseteq S_{0} \backslash\{0\}, w_{S_{0}, y}(T)=0 \leq w_{S_{0}, y}(S)$. Consider now $T_{0} \subseteq S_{0}$ and let $Q \subseteq M \backslash S_{0}$. Since $T_{0} \cap Q=\emptyset$,

$$
\kappa_{w}\left(T_{0} \cup Q\right)=\frac{w(M)-w\left(T_{0} \cup Q\right)}{m-\left|T_{0} \cup Q\right|+1}=\frac{w(M)-w\left(T_{0} \cup Q\right)}{m-t_{0}-q+1} .
$$

Moreover, $T_{0} \cup Q \subseteq M$ and $T_{0} \cup Q \ni 0$. By Step 1 of Algorithm 2, $\kappa_{w}\left(S_{0}\right)$ is the minimal among all coalitions in $M$ containing the veto-player 0 . From where and also because of the obvious inequality $s_{0}>t_{0}-1$, it holds that

$$
\frac{w(M)-w\left(T_{0} \cup Q\right)}{m-s_{0}-q}>\frac{w(M)-w\left(T_{0} \cup Q\right)}{m-t_{0}-q+1} \geq \kappa_{w}\left(S_{0}\right) .
$$

Hence,

$$
w(M)-\left(m-s_{0}\right) \cdot \kappa_{w}\left(S_{0}\right)>w\left(T_{0} \cup Q\right)-q \cdot \kappa_{w}\left(S_{0}\right),
$$

and therefore, since at Step 2 every player's $i \in M \backslash S_{0}$ payoff $y_{i}=\kappa_{w}\left(S_{0}\right)$, it holds that

$$
w(M)-y\left(M \backslash S_{0}\right)>w\left(T_{0} \cup Q\right)-y(Q) .
$$

Then by definition of the Davis-Maschler reduced game we obtain $w_{S_{0}, y}\left(S_{0}\right) \geq$ $w_{S_{0}, y}\left(T_{0}\right)$ for every $T_{0} \subseteq S_{0}$.

From the proof of Theorem 7 also the upper bound for the nucleolus payoff $\nu_{0}$ to the veto-player 0 in a veto-rich game $v^{0} \in \mathcal{G}_{N_{0}}^{+}$easily follows.

Proposition 3 For any veto-rich game $v^{0} \in \mathcal{G}_{N_{0}}^{+}$

$$
\nu_{0} \leq v^{0}\left(N_{0}\right)-n \cdot \kappa_{0}^{*}\left(v^{0}\right),
$$

with the equality, if and only if $\nu_{i}\left(v^{0}\right)=\nu_{j}\left(v^{0}\right)$ holds for all $i, j \in N$.

Proof Since $v^{0}(i)=0$ for all $i \in N$, every excess of a singleton coalition $\{i\}, i \neq 0$, with respect to the nucleolus $\nu\left(v^{0}\right)$ is equal to $-\nu_{i}\left(v^{0}\right)$. From (17) in the proof of 
Theorem 7 it follows that the maximal excess in $v^{0}$ with respect to the nucleolus is equal to $-\kappa_{0}^{*}\left(v^{0}\right)$. Therefore from the efficiency of the nucleolus we obtain that

$$
\nu_{0}=v^{0}\left(N_{0}\right)-\sum_{i \in N} \nu_{i}\left(v^{0}\right)=v^{0}\left(N_{0}\right)+\sum_{i \in N}\left(-\nu_{i}\left(v^{0}\right)\right) \leq v^{0}\left(N_{0}\right)+n \cdot\left(-\kappa_{0}^{*}\left(v^{0}\right)\right),
$$

where the equality hold, if and only if $\nu_{i}\left(v^{0}\right)=\kappa_{0}^{*}\left(v^{0}\right)$ for all $i, j \in N$.

Remark 5 The inequality (18) can be equivalently presented in the form

$$
\kappa_{0}^{*}\left(v^{0}\right) \leq \frac{v^{0}\left(N_{0}\right)-\nu_{0}}{n},
$$

with the equality, if and only if $\nu_{i}\left(v^{0}\right)=\nu_{j}\left(v^{0}\right)$ holds for all $i, j \in N$. Inequality (19) will be used later in the proof of Theorem 6 .

We are ready now to prove Theorem 6 .

Proof of Theorem 6 Consider a veto-removed game $v \in \mathcal{R}_{N}$. By definition of $\mathcal{R}_{N}$ there exists a monotonic veto-rich game $v^{0} \in \mathcal{G}_{N_{0}}^{m}$ such that $v$ is the Davis-Maschler reduced game of $v^{0}$ obtained by deleting the veto-player 0 in accordance to the nucleolus payoff. Then because of the already mentioned above the Davis-Maschler consistency of the nucleolus in a veto-rich game in $\mathcal{G}_{N_{0}}^{m}, \nu_{i}(v)=\nu_{i}\left(v^{0}\right)$ for all $i \in N$. Since Algorithm 2 yields $\nu\left(v^{0}\right)$, for proving Theorem 6 it is sufficient to show that the payoff vector $x$ produced by Algorithm 1 for the game $v$ coincides on $N$ with the payoff vector $y$ produced by Algorithm 2 for the game $v^{0}$. For giving evidence for that we show first that either in Step 1 of Algorithm $1 S=\emptyset$ is chosen and the algorithm yields the nucleolus, or it holds that

$$
\kappa^{*}(v)=\kappa_{0}^{*}\left(v^{0}\right) .
$$

From Theorem 7, $y=\nu\left(v^{0}\right)$. $v$ is the Davis-Maschler reduced game of $v^{0}$ obtained by deleting the veto-player 0 in accordance to the nucleolus $\nu\left(v^{0}\right)$. Hence by definition of the Davis-Maschler reduced game, $v(S)=\max \left\{0, v^{0}(S \cup\{0\})-y_{0}\right\}$, i.e., either $v(S)=v^{0}(S \cup\{0\})-y_{0}$ or $v(S)=0$. Let $S \varsubsetneqq N$ be the coalition chosen in Step 1 at the first iteration of Algorithm 1. It turns out that either $S=\emptyset$, or $v(S)=v^{0}(S \cup\{0\})-y_{0}$. Indeed if we assume that $S \neq \emptyset$ and $v(S)=0$, then

$$
\kappa_{v}(S) \stackrel{\text { def }}{=} \frac{v(N)-v(S)}{n-s+1}=\frac{v(N)}{n-s+1} \geq \frac{v(N)}{n} \stackrel{\text { def }}{=} \kappa_{v}(\emptyset)
$$

i.e., $\kappa_{v}(\emptyset) \leq \kappa_{v}(S)$ while $|\emptyset|=0<s$, which contradicts the choice of $S$.

Let now $S_{0} \varsubsetneqq N_{0}$ be the coalition chosen in Step 1 at the first iteration of Algorithm 2 and let $S=S_{0} \backslash\{0\}$. Similarly to the paragraph above, it turns out that either $S=\emptyset$, or $v(S)=v^{0}(S \cup\{0\})-y_{0}$. Indeed if $S \neq \emptyset$ and $v(S)=\max \left\{0, v^{0}(S \cup\right.$ $\left.\{0\})-y_{0}\right\}=0$, i.e., $v^{0}(S \cup\{0\})-y_{0}<0$, then

$$
\kappa_{v^{0}}\left(S_{0}\right) \stackrel{\text { def }}{=} \frac{v^{0}\left(N_{0}\right)-v^{0}\left(S_{0}\right)}{n_{0}-s_{0}+1}>\frac{v^{0}\left(N_{0}\right)-y_{0}}{n-s+1} \geq \frac{v^{0}\left(N_{0}\right)-y_{0}}{n},
$$

which contradicts Proposition 3 restated in the form (19). Thus, for the coalition $S_{0}$ chosen in Step 1 at the first iteration of Algorithm 2 it holds that either $S=$ $S_{0} \backslash\{0\}=\emptyset$, or $v(S)=v^{0}(S \cup\{0\})-y_{0}$. 
Hence, due to the Davis-Maschler reduced game relationship between $v$ and $v^{0}$, in both algorithms for all $S \varsubsetneqq N$ with the assumption that $S=S_{0} \backslash\{0\}$ for $S_{0}$ chosen in Step 1 of Algorithm 2, it holds that either $S=\emptyset$ or $v(S)=v^{0}(S \cup\{0\})-y_{0}$. Thus, for proving (20) it is sufficient to prove that for all $S \varsubsetneqq N$ it holds that $\kappa_{v}(S)=\kappa_{v^{0}}(S \cup\{0\})$.

First consider the case $S \varsubsetneqq N, S \neq \emptyset$. Then $v^{0}\left(S_{0}\right)=v(S)+y_{0}$, and in particular, $v^{0}\left(N_{0}\right)=v(N)+y_{0}$. Therefore,

$$
\kappa_{v^{0}}\left(S_{0}\right) \stackrel{\text { def }}{=} \frac{v^{0}\left(N_{0}\right)-v^{0}\left(S_{0}\right)}{n_{0}-s_{0}+1}=\frac{v(N)-v(S)}{n-s+1} \stackrel{\text { def }}{=} \kappa_{v}(S)
$$

i.e.,

$$
\kappa_{v^{0}}\left(S_{0}\right)=\kappa_{v}(S), \quad \text { for all } S \varsubsetneqq N, S \neq \emptyset .
$$

Consider now the case $S=\emptyset$. Then $S_{0}=S \cup\{0\}=\{0\}$ and

$$
\kappa_{v^{0}}\left(S_{0}\right)=\kappa_{v^{0}}(\{0\}) \stackrel{\text { def }}{=} \frac{v^{0}\left(N_{0}\right)-v^{0}(\{0\})}{n_{0}} .
$$

Again there are two options possible, namely $\kappa_{v^{0}}(\{0\})=\kappa_{0}^{*}\left(v^{0}\right)$ or $\kappa_{v^{0}}(\{0\})>$ $\kappa_{0}^{*}\left(v^{0}\right)$.

If $\kappa_{v^{0}}(\{0\})=\kappa_{0}^{*}\left(v^{0}\right)$, then Algorithm 2 terminates at the first iteration and in Step 2 every player $i \in N$ gets the same payoff $y_{i}=\kappa_{v^{0}}(\{0\})$. Due to the coincidence of nucleoli in games $v$ and $v^{0}$ on $N$, it holds that every player $i \in N$ in $v$ has the same nucleolus payoff that by efficiency is equal to $\frac{v(N)}{n}$. Hence, with $i \in N$,

$$
\kappa_{v^{0}}(\{0\})=y_{i} \stackrel{\text { eff }}{=} \frac{v(N)}{n} \stackrel{\text { def }}{=} \kappa_{v}(\emptyset)
$$

From where together with $(21)$ it follows that $(20)$ holds true when $\kappa_{v^{0}}(\{0\})=$ $\kappa_{0}^{*}\left(v^{0}\right)$.

If $\kappa_{v^{0}}(\{0\})>\kappa_{0}^{*}\left(v^{0}\right)$, then there exists $S \varsubsetneqq N, S \neq \emptyset$, such that $\kappa_{v^{0}}\left(S_{0}\right)=\kappa_{0}^{*}\left(v^{0}\right)$. Because of $(21), \kappa_{v^{0}}\left(S_{0}\right)=\kappa_{v}(S)$, and hence,

$$
\kappa_{v}(S)=\kappa_{0}^{*}\left(v^{0}\right) \stackrel{(19)}{\leq} \frac{v^{0}\left(N_{0}\right)-y_{0}}{n}=\frac{v(N)}{n} \stackrel{\text { def }}{=} \kappa_{v}(\emptyset),
$$

where the second equality is due to $v$ being the Davis-Maschler reduced game of $v^{0}$. Whence, either $\kappa_{v}(\emptyset)=\kappa_{v}(S)$, or $\kappa_{v}(\emptyset)>\kappa_{v}(S)$.

If $\kappa_{v}(\emptyset)=\kappa_{v}(S)$, then

$$
\kappa_{0}^{*}\left(v^{0}\right)=\frac{v^{0}\left(N_{0}\right)-y_{0}}{n},
$$

and from Proposition 3 and Remark 5 it follows that $\nu_{i}\left(v^{0}\right)=\nu_{j}\left(v^{0}\right)$ for all $i, j \in N$, and therefore, for all $i \in N$,

$$
\nu_{i}\left(v^{0}\right) \stackrel{\mathrm{D}-\mathrm{M} \text { concist }}{=} \nu_{i}(v) \stackrel{\text { eff }}{=} \frac{v(N)}{n} \stackrel{\text { def }}{=} \kappa_{v}(\emptyset),
$$

i.e., in Step 1 of Algorithm 1 the empty set is chosen and Algorithm 1 in fact yields the nucleolus. 
If $\kappa_{v}(\emptyset)>\kappa_{v}(S)$, then there exists $S^{\prime} \varsubsetneqq N, S^{\prime} \neq \emptyset$, such that $\kappa_{v}\left(S^{\prime}\right)=\kappa^{*}(v)$ (possibly, $S^{\prime}=S$ ). Hence, due to $(21)$, for $S_{0}^{\prime}=S^{\prime} \cup\{0\} \varsubsetneqq N_{0}, \kappa_{v}\left(S^{\prime}\right)=\kappa_{v^{0}}\left(S_{0}^{\prime}\right)$, i.e., in this case $\kappa^{*}(v)=\kappa_{0}^{*}\left(v^{0}\right)$ as well. Thus, it is proved that either in Step 1 of Algorithm 1 either $S=\emptyset$ is chosen and the algorithm yields the nucleolus, or $\kappa^{*}(v)=\kappa_{0}^{*}\left(v^{0}\right)$.

For completing the proof it remains to consider the situation when in Step 1 of Algorithm 1 a coalition $S \varsubsetneqq N, S \neq \emptyset$, is chosen. As it is shown above in such a case in Step 1 of Algorithm 2 we always can chose $S_{0} \varsubsetneqq N_{0}, S_{0}=S \cup\{0\}$, for which $\kappa_{v^{0}}\left(S_{0}\right)=\kappa_{v}(S)$. Thus, at the first iteration both algorithms at Step 2 assign $x_{i}=y_{i}$ for every $i \in N \backslash S=N_{0} \backslash S_{0}$. It is easy to see that the Davis-Maschler reduced game $w_{S, x}$ constructed in Step 3 of Algorithm 1 is the Davis-Maschler reduced game of the Davis-Maschler reduced game $w_{S_{0}, y}$ constructed in Step 3 of Algorithm 2. Then observe that the situation at all next iterations of both algorithms remains the same. Therefore, repeating the same reasoning as above we obtain that both algorithms assign the same payoffs to all players in $N$.

\section{References}

[1] Arin, J., V. Feltkamp (1997), The nucleolus and kernel of veto-rich transfereble utility games, International Journal of Game Theory, 26, 61-73.

[2] Aumann, R.J., M. Maschler (1985), Game theoretic analysis of a bankruptcy problem from the Talmud, Journal of Economic Theory, 36, 195-213.

[3] Davis, M., M. Maschler (1965), The kernel of a cooperative game, Naval Research Logistics Quarterly, 12, 223-259.

[4] Deprez, O., H.U. Gerber (1985), On convex principle of premium calculation, Insurance: Mathematics and Economics, bf 4, 179-189.

[5] Driessen, T.S.H. (1985), Properties of 1-convex n-person games, OR Spektrum, 7, 19-26.

[6] Driessen, T.S.H., A.B. Khmelnitskaya, J. Sales (2005), 1-concave basis for TU games, Memorandum No. 1777, Department of Applied Mathematics, University of Twente, The Netherlands.

[7] Driessen, T.S.H., S.H. Tijs (1983), The $\tau$-value, the nucleolus and the core for a subclass of games, Methods of Operations Research, 46, 395-406.

[8] Fragnelli, V. M.E. Marina (2004), Co-Insurance Games and Environmental Pollution Risk, in: Carraro C, Fragnelli V (eds.) Game Practice and the Environment, Edward Elgar Publishing, Cheltenham (UK), pp. 145-163.

[9] Gillies, D.B. (1953), Some theorems on $n$-person games, Ph.D. thesis, Princeton University.

[10] Kohlberg, E. (1971), On the nucleolus of a characteristic function game, SIAM Journal on Applied Mathematics, 20, 62-66. 
[11] Quant, M., P. Borm, H. Reijnierse, B. van Velzen (2005), The core cover in relation to the nucleolus and the Weber set, International Journal of Game Theory, 33, 491-503.

[12] Schmeidler, D. (1969), The nucleolus of a characteristic function game, SIAM Journal on Applied Mathematics, 17, 1163-1170.

[13] Shapley, L.S. (1971), Cores of convex games, International Journal of Game Theory, 1, 11-26.

[14] Sobolev, A.I. (1975), The characterization of optimality principles in cooperative games by functional equations, in: Vorob'ev N.N. (ed.) Mathematical Methords in the Social Sciences, 6, Vilnus, pp. 94-151 (in Russian). 